$\tilde{g}_{86}=10^{27} \mathrm{sec}^{-2}$ is taken.

With these values, $T_{c}(8.75 \mathrm{kV} / \mathrm{cm})-T_{c}(0)$

$\simeq 2.5{ }^{\circ} \mathrm{C}$, which is close to the experimentally de-

termined difference.

This supports the hypothesis of the predominant influence of the electric field on $T_{c}$. In fact, the calculation of the displacement of the transition temperature $T_{c}$ corresponds to that of the secondorder phase transition only. In the case of GMO, the sixth-order terms must be introduced in $F$ to compute the field effect on the transition temperature.
The coefficient $\beta$ has already been measured in some crystals. In quartz, $\beta \simeq 3 N^{-1} \mathrm{~m}^{-1},{ }^{11}$ and in $\mathrm{LiNbO}_{3}, \beta \simeq 35 N V^{-1} \mathrm{~m}^{-1} .12$ These values may be compared to $2.5 \mathrm{~N} \mathrm{~V}^{-1} \mathrm{~m}^{-1}$ in GMO far removed from the transition, but are 5-50 times smaller than values of $\beta$ measured here near the transition.

In conclusion, this paper presents one of the first direct measurements of the critical behavior of a third-order coupling coefficient between electric field and strain in the vicinity of the ferroelasticferroelectric phase transition.

*Associated with the Centre National de la Recherche Scientifique.

${ }^{1} \mathrm{H}$. J. Borchardt and T. E. Bierstedt, Appl. Phys. Lett. 8,50 (1966).

${ }^{2} \bar{K}$. Aizu, A. Kumada, H. Yumoto, and S. Ashida, J. Phys. Soc. Jap. 27, 511 (1969).

${ }^{3} \mathrm{~J}$. Kobayashi, Y. Sato, and T. Nakamura, Phys. Status Solidi A 14, 259 (1972).

${ }^{4}$ L. E. Cross, A. Fouskova, and S. E. Cummins, Phys. Rev. Lett. 21, 812 (1968).

${ }^{5}$ C. Scheiding and G. Schmidt, Phys. Status Solidi B 53, K95 (1972).

${ }^{6}$ V. T. Höchli, Phys. Rev. B 6, 1814 (1972).

${ }^{7}$ V. Dvorak, and J. Petzelt, Phys. Lett. A 35, 209

(1971).

${ }^{8}$ In this paper, all the expressions where the tilde is used for the coefficients are written in the tetragonal axes. Without the tilde, the expressions are written in the orthorhombic axes.

${ }^{9}$ J. D. Axe, B. Dorner, and G. Shirane, Phys. Rev. Lett. 26,519 (1971).

${ }^{10} \mathrm{~J}$. M. Courdille and J. Dumas, Solid State Commun. 9,609 (1971).

${ }^{11} \overline{\mathrm{K}}$. Hruska, and V. Kazda, Czech. J. Phys. B $\underline{18}, 500$ (1968).

${ }^{12}$ R. B. Thompson and C. S. Quate, J. Appl. Phys. $\underline{42}$, 907 (1971).

\title{
Measurement of a Critical Exponent by a New Zero-Field Transmission Resonance Technique*
}

\author{
C. N. Manikopoulos, $†$ P. Sheng, $\ddagger$ and T. R. Carver \\ Joseph Henry Laboratories of Physics, Princeton University, Princeton, New Jersey 08540 \\ (Received 10 January 1973)
}

The critical exponent $\beta$ of Gd is measured directly for the first time by a new technique of ferromagnetic transmission resonance in zero external field. The technique consists of measuring the microwave power transmitted through a thin Gd film $(47 \mu)$ as a function of temperature. Analysis of the resulting line shape yields the value of conductivity $\sigma=0.745 \times 10^{16} \mathrm{sec}^{-1}$, spin relaxation time $\tau=7.3 \times 10^{-11} \mathrm{sec}$, Curie temperature $T_{C}=295^{\circ} \mathrm{K}$, and critical exponent $\beta=0.31 \pm 0.05$.

\section{INTRODUCTION}

Critical-point phenomena have been studied with renewed interest in recent years, partly because of stimulating new theoretical work on statistical mechanics, ${ }^{1}$ scaling, ${ }^{2}$ and controversial questions as to the uniqueness of some of the parameters such as the critical exponents, ${ }^{3}$ and partly because of vast improvements in the ability to measure such exponents. Among the most accurate of these experimental techniques are nuclear resonance in antiferromagnets, ${ }^{4}$ and critical opalescence measured by laser techniques in binary liquids. ${ }^{5}$ Mössbauer-effect measurements are also important in a few substances. ${ }^{5}$ However, experiments to measure the critical exponents in ferromagnetic metals have not been as accurate because of strong damping effects and large linewidths. Typically, accuracies of $5-15 \%$ in the latter case are contrasted with accuracies of nearly $1 \%$ in the more precise determinations.

In this paper we describe a measurement of the coefficient $\beta$ for gadolinium defined by

$$
M(T) / M(0)=B\left(1-T / T_{C}\right)^{\beta}, \quad T \rightarrow T_{C} .
$$

This measurement is distinguished by being car ried out by means of a new technique, possibly applicable to other ferromagnets such as iron and 
nickel, and by the fact that it is the first direct determination of this coefficient for gadolinium, even though a value for $\beta$ has been given in the literature by indirect fitting of magnetization through the scaled equation of state. ${ }^{6}$ The measurement is less distinguished in accuracy, however, and does not yet approach the $1 \%$ accuracy previously mentioned.

Our new technique involves the observation of a zero-field resonance in a thin single crystal of gadolinium by means of the microwave-resonance transmission technique. Since it does involve a zero $H$ field, one must sweep through the resonance by varying the temperature instead of the field and this determines $M(T)$ through interpretation of the resulting line shape.

To make a simple introduction to the subject, we first briefly review the nature of spin transmission resonance. The method was originally developed to make the observation of conduction-electronspin resonance in metals easier and less ambiguous. ${ }^{7}$ A thin sample, thicker, however, than the skin depth, is used as a common wall between two microwave cavities. When placed in a magnetic field suitable for electron resonance, a microwave signal in one cavity induces resonant spin precession and the spins, which diffuse across the sample from the skin depth region of one cavity to the other, radiate a resonance signal into the second cavity connected to a microwave receiver with a good noise figure. The finite thickness of the sample means that only specific modes rather than impurity resonances will be observed, and the fact that exciting power is not present in the second cavity leads to excellent sensitivity for reception.

This technique was soon found to be useful in detecting ferromagnetic "antiresonance" or paramagnetic-dispersion skin-depth enhancement, as it might also be called. ${ }^{8}$ In this mode, even though the spins are not mobile and do not diffuse through the sample, the presence of a resonant condition leads to dispersion, which, in turn, causes a change in the skin depth. That is, the skin depth depends on the permeability of the sample as well as its conductivity. Another way of explaining the process, as will be discussed later, is to say that a condition occurs when the coupling between the magnetic system of the spins, described by the Bloch equation, and the conduction-electron cur rents, described by Maxwell's equations, leads to magnetic energy storage in the spin system. This reduces dissipation and increases the skin depth. Though the effect is small in a typical sample many skin depths thick, it corresponds to a trans mitted power increase from $10^{-16}$ to $10^{-12}-10^{-14} \mathrm{~W}$, which is readily detectable.

Of particular importance to the present mea- surement is the fact that this type of propagated signal occurs at a condition of maximum in the field $B$ which matches the resonant condition $\omega_{0}$ $=\gamma_{e} B$, where $\gamma_{e}$ is the gyromagnetic ratio of the spin system and $\omega_{0}$ is the microwave frequency, in contrast to the usual condition for the maximum of surface impedance ferromagnetic absorption which occurs, apart from demagnetization effects, as the usual resonance condition $\omega_{0}=\gamma_{e} H$. Thus a condition may be found in the complete absence of any applied magnetic field that $\omega_{0}=\gamma_{e} 4 \pi M .^{9}$ The fact that $M$ is a function of temperature allows one to sweep through resonance as one sweeps through the critical Curie point $T_{C}$. Interpretation of the signal must be done by recourse to theory, but the experiment is relatively straightforward. Difficulties arise in ascertaining the effects of anisotropy, in determining the temperature scale, in making sure that the cavities in the apparatus are properly adjusted at all temperatures, and making sure of proper crystal alignment in the sample. All these aspects will be discussed in the body of the article and the appendices.

\section{THEORY}

Suppose we have a ferromagnetic single crystal in the form of a film of thickness $l$, with its surface lying in the $x z$ plane. The magnetization $M$ is taken to be along the $z$ axis, and the applied microwaves are assumed to be plane waves normally incident upon the $x z$ plane, the tangential component of the magnetic vector being along the $x$ axis (see Fig. 1). In this section we will be concerned only with the transmission of electromagnetic waves through the sample. The effects of exchange interaction, anisotropy energy, and magnetostriction are analyzed in the appendices and can be shown to be small.

Calculation of the ratio of the transmitted magnetic vector to the incident magnetic vector, $h_{3} / h_{1}$, proceeds in two steps: (i) calculate the wave vector $k_{2}$ inside the sample as a function of frequency $\omega$, and (ii) fit boundary conditions to obtain the desired expression for $h_{3} / h_{1}$. One needs only Maxwell's equations (which describe the radiation field) and Bloch's phenomenological equation (which describes the interaction between the radiation field and the magnetization):

$$
\begin{aligned}
& \nabla \times \overrightarrow{\mathrm{E}}=-\frac{1}{c} \frac{\partial}{\partial t}(\overrightarrow{\mathrm{H}}+4 \pi \overrightarrow{\mathrm{M}}), \\
& \nabla \times \overrightarrow{\mathrm{H}}=4 \pi \sigma \overrightarrow{\mathrm{E}} / c, \\
& \frac{\partial \overrightarrow{\mathrm{M}}}{\partial t}=\gamma_{e} \overrightarrow{\mathrm{M}} \times \overrightarrow{\mathrm{H}}-(\overrightarrow{\mathrm{M}}) \text { transverse } / \tau .
\end{aligned}
$$

Here $\gamma_{e}\left(=1.72 \times 10^{7} \mathrm{~Hz} / \mathrm{G} \text { for } \mathrm{Gd}\right)^{10}$ is the gyromagnetic ratio, $c$ the speed of light, $\sigma$ the conductivity, $\tau$ the phenomenological spin relaxation time 
(typically $10^{-10} \mathrm{sec}$ ), ${ }^{11} \overrightarrow{\mathrm{M}}$ the magnetization vector, and $\vec{E}$ and $\overrightarrow{\mathrm{H}}$ the electric and magnetic field vectors, respectively. ${ }^{12}$

Taking the curl of Eq. (2), one can eliminate $\overrightarrow{\mathrm{E}}$ :

$$
\nabla(\nabla \cdot \overrightarrow{\mathrm{H}})-\nabla^{2} \overrightarrow{\mathrm{H}}=-\frac{4 \pi \sigma}{c^{2}} \frac{\partial}{\partial t}(\overrightarrow{\mathrm{H}}+4 \pi \overrightarrow{\mathrm{M}}) .
$$

Since the microwave magnetic field will be much smaller in magnitude than the $z$ component of magnetization, the disturbance of the magnetization caused by the microwave field will be in the $x y$ plane, and the change in $z$ component of magnetization will only be of second order. Therefore, let

$$
\begin{aligned}
& \mathbf{M}=m_{x} \hat{i}+m_{y} \hat{j}+M \hat{k}, \\
& \overrightarrow{\mathrm{H}}=h_{x} \hat{i}+h_{y} \hat{j},
\end{aligned}
$$

where the components $m_{x}, m_{y}, h_{x}$, and $h_{y}$ are understood to be proportional to $e^{i\left(k_{2} y-\omega t\right)}$. When

(5a) and (5b) are substituted into (3) and (4), these equations, in their component form, become

$$
\begin{aligned}
& \left(k_{2}^{2}-i \frac{4 \pi \sigma \omega}{c^{2}}\right) h_{x}-i \frac{16 \pi^{2} \sigma \omega}{c^{2}} m_{x}=0, \\
& h_{y}=-4 \pi m_{y}, \\
& -i \omega m_{x}=4 \pi M_{e} m_{y}-m_{x} / \tau, \\
& -i \omega m_{y}=\gamma_{e} M h_{x}-m_{y} / \tau .
\end{aligned}
$$

In order that there be a nontrivial solution, the determinant of the coefficients must vanish. This condition gives

$$
k_{2}^{2}=\left(2 i / \delta^{2}\right)\left[1+R^{2} /(\lambda-i \Omega)^{2}\right],
$$

where

$$
\begin{array}{ll}
\delta^{2}=c^{2} / 2 \pi \sigma \omega, & R=M(T) / M(0), \\
\lambda=1 / 4 \pi M(0) \gamma_{e} \tau, & \Omega=\omega / 4 \pi M(0) \gamma_{e} .
\end{array}
$$

One should note that for frequencies much higher than $4 \pi M(0) \gamma_{e}\left(=4.43 \times 10^{11} \mathrm{rad} / \mathrm{sec}\right.$ for $\left.\mathrm{Gd}\right)$ the solution (7) becomes identical with the usual skindepth solution $k_{2}=(1+i) / \delta$; and for slow spin relaxation $(\lambda \ll 1), k_{2}$ goes to zero as $\omega$ approaches $4 \pi M(T) \gamma_{e}$. We can get a physical picture of the interactions between the electromagnetic field and the spin system by looking at the relations between $h_{x}, h_{y}, m_{x}$, and $m_{y}$.

$$
\begin{aligned}
& \text { From Eqs. (6b) }-(6 \mathrm{~d}) \text {, we get } \\
& R^{2} /(\lambda-i \Omega)^{2} h_{x}=4 \pi m_{x}, \\
& h_{y}=-4 \pi m_{y}, \\
& (\lambda-i \Omega) m_{x}=R m_{y} .
\end{aligned}
$$

If $\lambda \ll 1$, we see that at $\omega \simeq 4 \pi M(T) \gamma_{e}, R=\Omega$, so that $h_{y}=-4 \pi m_{y}$ and $h_{x} \simeq-4 \pi m_{x}$. In other words, at $\omega \simeq 4 \pi M(T) \gamma_{e}, b_{x, y}=h_{x, y}+4 \pi m_{x, y} \simeq 0$. As we see, $b_{x, y} \simeq 0$ implies $(i)-\vec{m} \cdot \vec{h}=$ (energy of the spin system) is maximum and (ii) $\nabla \times \vec{E}=0$ from Eq. (1) means that either $\vec{E}=0$ or $\vec{E}$ is uniform across the sample. It turns out that $\overrightarrow{\mathrm{E}} \simeq 0$ for $\omega \simeq 4 \pi M(T) \gamma_{e}$ is the electric field configuration inside an infinitely thick plane and that uniform $\vec{E}$ is the configuration inside a film of finite thickness. In order to be more specific, we proceed to fit the boundary conditions. These state that the parallel components of $\vec{h}$ and $\vec{e}$ are continuous across the sample boundary. A simple calculation yields

$$
\frac{h_{3}}{h_{1}}=-i \frac{2 \omega \delta^{2}}{c} \frac{k_{2}}{f_{-}-f_{+}},
$$

where

$$
f_{ \pm}=\left(1 \pm i \frac{k_{2} \delta^{2} \omega}{2 c}\right)^{2} e^{ \pm i k_{2} l}
$$

The quantity experimentally observed is $\left|h_{3} / h_{1}\right|$. Here | | denotes the magnitude of $h_{3} / h_{1}$ 。 It should be noted that the expression for $\left|h_{3} / h_{1}\right|$ has a maximum at $\omega \simeq 4 \pi M(T) \gamma_{e}$. Near the maximum the electric and magnetic fields have the following configurations inside the sample if $\lambda=0$ :

$$
\begin{aligned}
& e_{x} \simeq(c / 4 \pi \sigma)\left(2 h_{1 x} / l\right), \\
& h_{x} \simeq 2 h_{1 x}\left(1-y / l^{\prime}\right),
\end{aligned}
$$

where

$$
l^{\prime}=l+\omega \delta^{2} / 2 c .
$$

Here $h_{1 x}$ is the incident magnetic field vector and $y$ is the distance from the incident surface. The energy flow, which is proportional to $\vec{e} \times \vec{h}$, decreases linearly as a function of distance inside the sample. The physics is therefore very clear: because part of the energy is stored in the spin system, only that part of the energy contained in the electromagnetic fields will be dissipated (since energy dissipation is proportional to $E^{2}$ ). As energy is transported through the sample, the energy in the spin system will decrease, but only linearly. This linear attenuation, as contrasted with the usual exponential attenuation, gives rise to an enhanced transmission. In this context the frequency $4 \pi M(T) \gamma_{\theta}$ is physically that frequency at which the energy of the spin system is maximum.

\section{EXPERIMENTAL APPARATUS AND TECHNIQUE}

\section{A. Microwave Apparatus}

The microwave-transmission apparatus used in this work is identical to the one used to observe conduction-electron-spin resonance, and has already been discussed in detail elsewhere. ${ }^{11,13}$ The apparatus consists of two microwave cavities, one the exciting cavity and the other the receiving cavity, with the sample forming the common wall between them. A superheterodyne receiver is attached to the receiving cavity. The only addition 


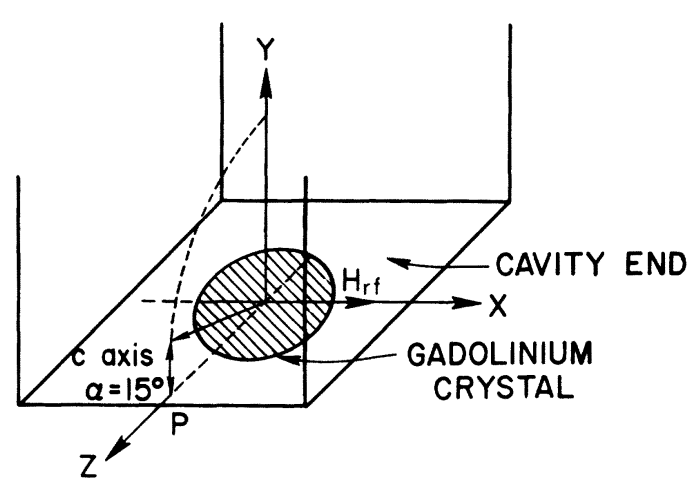

FIG. 1. Sample orientation in the cavity.

to the apparatus in the present work is a continuously variable motor-driven $360^{\circ}$ phase shifter at the output of the local oscillator which allows us to measure the transmitted microwaye signal power rather than the phase-dependent amplitude conventionally detected. Phase-independent power measurements have often been used before by other workers in the field. ${ }^{14}$ It is best suited for this work since it eliminates the phase parameter while there is still ample detail available in the powermeasurement line shape to allow determination of the other parameters involved in the problem. Moreover, in this type of experiment it would be experimentally impossible to obtain meaningful phase information as a function of temperature since the cavities detune significantly with temperature and must be retuned before the power measurement at each temperature reading.

\section{B. Sample Preparation}

The sample used was cut from an extremely pure single-crystal gadolinium button of size $\frac{1}{4} \times \frac{1}{4} \times \frac{1}{8}$ in. generously provided to us by Spedding. ${ }^{15}$ In order to cut thin slices from the crystal it was set in clear epoxy inside a boat mounted on a magnetic steel slab. The slab was then solidly locked onto a magnetic table beneath a 0.016-in. diamond disk saw. The cuts were taken along the broad side of the crystal with successive passes each about 250- $\mu$ thick. To further reduce the thickness of the slices we used a chemical lapping technique kindly suggested to us by Schmidt. ${ }^{16}$ The samples were mounted with wax on a special high-precision machined stainless-steel lapping holder which could expose the crystal at steps of $25 \mu$ to an acid surface of $1: 1$ nitric acid and acetic acid. The acid mixture was poured onto a Teflon cloth laid on a flat glass table. We then lapped by hand, using a figure-eight motion on the acid-wet cloth, for about $10 \mathrm{sec}$ at a time, quickly rinsing with running water after each lapping session. Both sides of the crystal samples were lapped to make sure that all damaged layers of the crystal were removed. We aimed to reduce the foils down to thickness between 25 and $50 \mu$. The thickness of each sample was established by measuring its surface area and its weight. The specific sample with which the data were taken was $47 \mu$.

Subsequently, the crystal was mounted on a goniometer and oriented by $x$-ray crystallography techniques. The $c$ axis of the crystal sample was found to be inclined to the surface at an angle $\alpha$ $=15^{\circ}$ with accuracy better than $1^{\circ}$, as shown on Fig. 1. X-ray pictures also verified that the sample was indeed a single crystal over the entire face exposed to the microwaves. Figures 1-3 together indicate the manner in which the sample was mounted with conducting indium seals between the two cavities.

\section{Temperature Measurement and Control}

To control the temperature of the sample a conventional Wheatstone-bridge circuit was used: a $10 K$ thermistor mounted on the cavity block as the control element, a $10 \mathrm{~K}$ helipot as the adjustable resistor set to the corresponding temperature desired, and two $10 \mathrm{~K}$ precision resistors were used as reference standards. When the cavity-block temperature is lower than the desired temperature the bridge unbalances and its output turns on a zero-voltage-crossing switch (RCA 3059) which provides a gate pulse to trigger a triac (GE MAC 2). The gate turns off when the cavity temperature becomes higher than the set temperature. The triac feeds about $5 \mathrm{~W}$ to three power resistors mounted on the cavity block. We can thus suppress temperature drifts of the cavity system to less than $50 \mathrm{~m}^{\circ} \mathrm{K}$ as determined by monitoring an iron-constantan thermocouple soldered on the cavity block.

Two more iron-constantan thermocouples ( 1 and

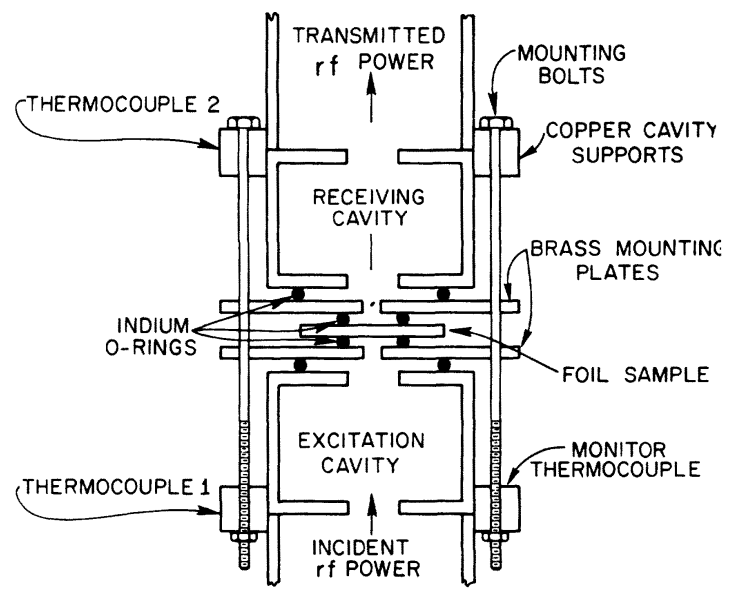

FIG. 2. Sample mounting and thermometry. 


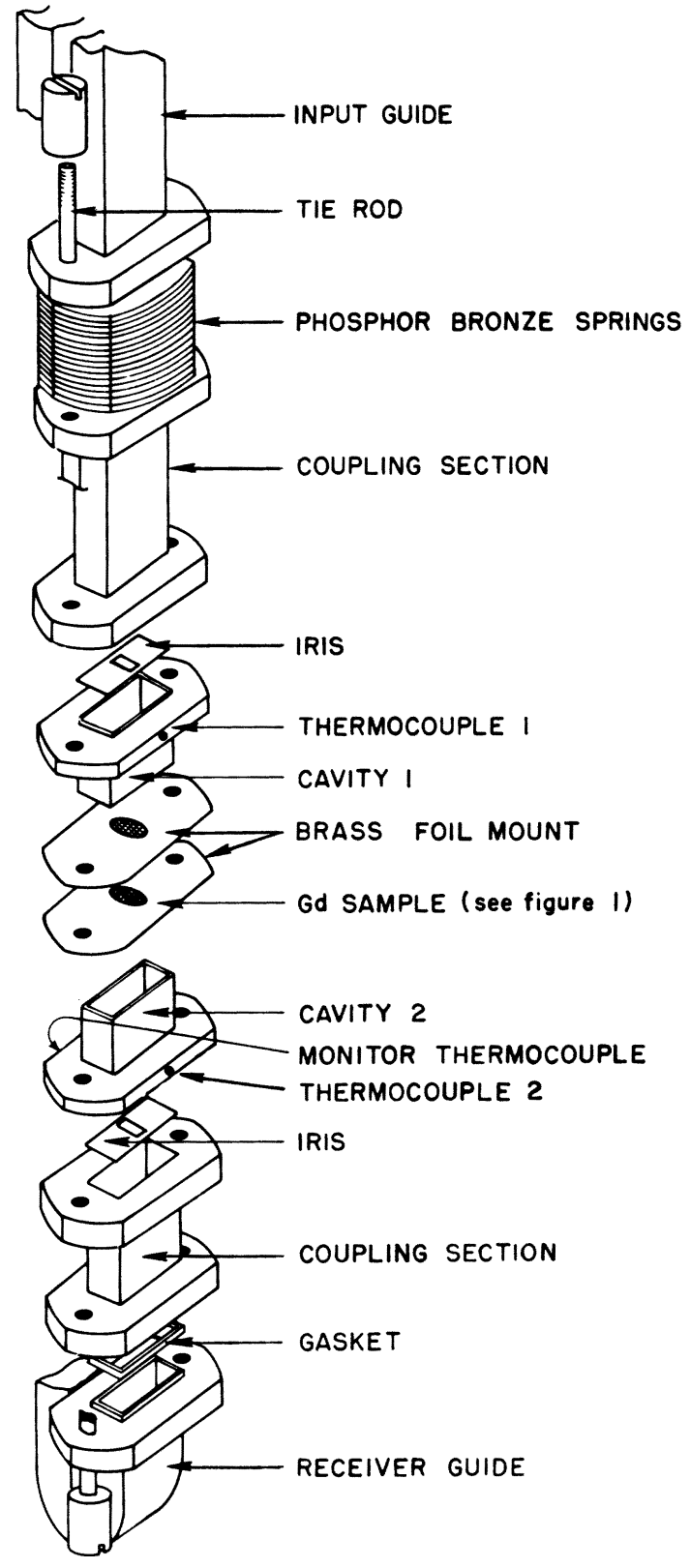

FIG. 3. Cavity assembly.

2) measure the temperatures of the exciting and receiving cavity, respectively (see Figs. 2 and 3 ). We observed that the exciting cavity ran hot with respect to the receiving cavity by as much as $1^{\circ} \mathrm{K}$. This is hardly surprising since the exciting cavity consumes about $300 \mathrm{~mW}$ of incident rf power. Since power dissipation is proportional to the area and to the square root of resistivity of the dissipating surface only a small fraction of the microwave power in the exciting cavity is dissipated at the sample. We find that the actual power dissipated at the sample is about $2 \mathrm{~mW}$. This implies that the temperature gradient across the sample is of the order of our temperature-control error which is thus the dominant limitation in our knowledge of the temperature of the sample.

Since the indium O-ring seals provide excellent electrical but poor thermal conductivity, the sample is only weakly thermally coupled to either cavity. Since the temperature of the sample is expected to be somewhere between that of the two cavities, we use the mean of the temperature of the two cavities as the actual sample temperature. Hence absolute sample-temperature measurements suffer by the order of the temperature difference of the two cavities. Relative temperature measurements, i. e., temperature differences, are not affected since thermal drifts were observed to be no more than $50 \mathrm{~m}^{\circ} \mathrm{K}$. Extraction of the critical exponent $\beta$ is sensitive only to the difference $T-T_{C}$ $=\Delta T$ once $T_{C}$ has been chosen, and not to the absolute value of $T$. On the other hand the experimentally observed critical temperature $T_{c}$ can be raised by about the order of $1^{\circ} \mathrm{K}$. This can explain our high value of $T_{C}$ in comparison to the results of other authors on similar samples ${ }^{17,18}$ which are listed as $292.5 \pm 0.5^{\circ} \mathrm{K}$ in contrast to our value of $295^{\circ} \mathrm{K}$. But it is interesting to note also the high value of $T_{C}=298^{\circ} \mathrm{K}$ observed by Darnell in measuring crystal lattice parameters in gadolinium. ${ }^{19}$

\section{Power Measurements}

To understand the meaning of our experimental points we refer to the schematic diagram of Fig. 4, which roughly presents the important functions of the microwave transmission apparatus. The incident microwave power $P$ from the klystron arrives at the switch $W$ which can direct the microwave power either through arm 1 or arm 2. In arm 1 the microwave power $P$ is attenuated by the $170-\mathrm{dB}$ calibrated attenuator $A$ to the value $P A$ and arrives at the detector $D$ which reads a level $B=P A D$ where $D$ is the detector sensitivity. Similarly in arm 2 the microwave power goes through the cavity system $C$ and then to the same detector $D$ which

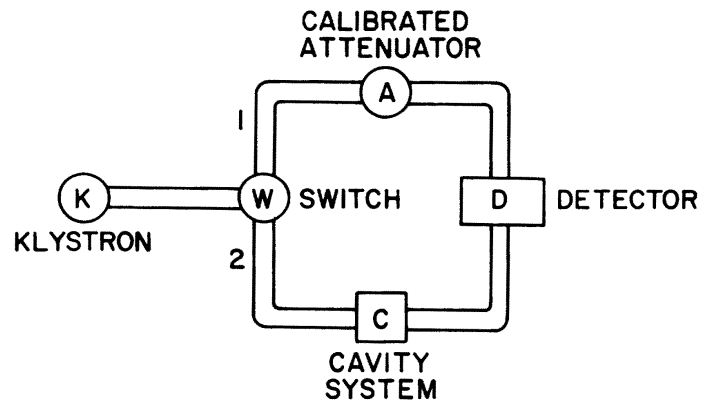

FIG. 4. Power measurement schematic. 
now reads a level $S=P C D$; here $C$ is the effective attenuation presented by the cavity-sample system to the microwave power and can be written as $C$ $=C_{E} C_{R} T$, where $C_{E}$ and $C_{R}$ are the coupling power coefficients for the exciting and receiving cavities, respectively, and $F$ expresses the attenuation of the microwave power in propagating through the sample. At each temperature after tuning the cavities we measure the calibration level $B$ and then immediately measure the signal level $S$. The experimental points $R$ are then defined as $R=S / B=P C D /$ $P A D=C / A$ and are thus independent of long-term klystron-power-level and detector-sensitivity drifts. The theoretical signal expressions can be directly related to the attenuation coefficient $F$ which can be written as $F=C / C_{E} C_{R}=R A / C_{E} C_{R}$, so that for constant attenuation setting $A$ and coupling coefficients $C_{E}$ and $C_{R}, F$ is directly proportional to just $R$. The experimental data show $R$ or, equivalently,
$F$ as a function of temperature. The experimental value of $C_{E} C_{R}$ is 0.85 .

\section{INTERPRETATION}

We note that in Fig. 5 the data on transmitted power agree in general shape with that calculated from the theory. The line shape as a function of temperature consists of three parts. Above the Curie temperature the transmitted power is a slow varying function of temperature. We call this region I. As temperature is lowered through $T_{C}$ the transmitted power increases within one or two degrees (region II) to a peak and then slowly decreases as the temperature is further reduced (region III). In order to fit the data, we will proceed in three steps. The first step is to note that in Eq. (8) when $M=0, k_{2}$ reduces to $(1+i) / \delta$, which is the usual skin-depth attenuation. Therefore, from the data in region $\mathrm{I}$, thickness $l$ of the film, and fre-

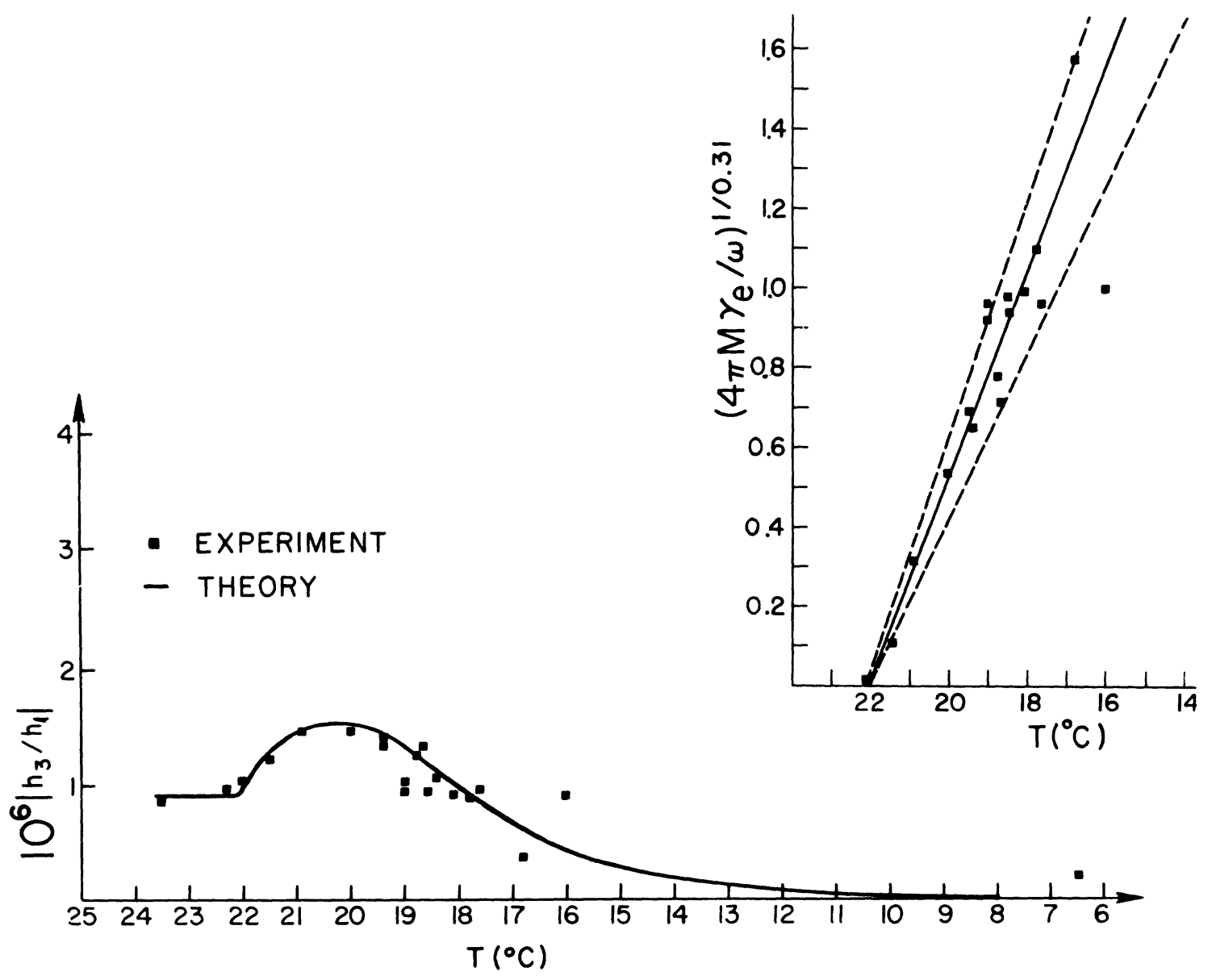

FIG. 5. $\left|h_{3} / h_{1}\right|$ plotted as a function of temperature $T$. The inset shows $\left[4 \pi M(T) \gamma_{e} / \omega\right]^{1 / 0.31}$ plotted as a function of $T$. $M(T)$ is obtained from $\left|h_{3} / h_{1}\right|$ through Eq. (8). Dashed lines indicate the slopes from which the credible limits of $B$ are obtained. 

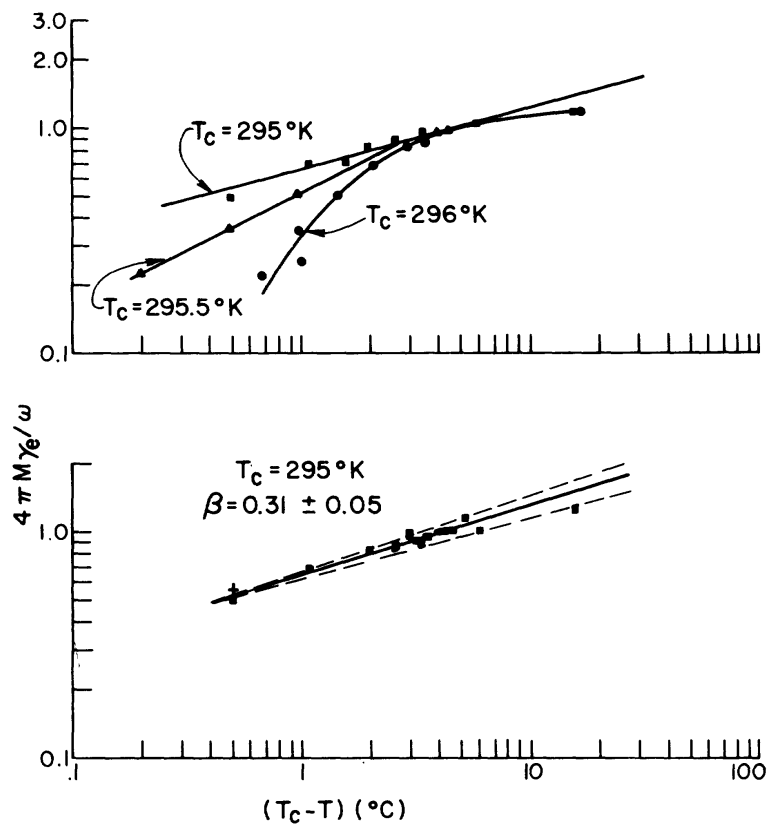

FIG. 6. $4 \pi M(T) \gamma_{e} / \omega$ plotted as a function of $\left(T_{C}-T\right)$. The upper figure shows the effect of choosing different values of $T_{C}$. The lower figure shows the best fit to the data. Dashed lines indicate the credible limits of the value $\beta$. Owing to crowding, not all the data points are displayed in the upper figure.

quency $\omega$ of the microwave radiation, we can extract the value of conductivity $\sigma$. Since the electric vector is along the $c$ axis in the present experiment (see Fig. 1), this value of $\sigma$ should be that along the $c$ axis. Using the experimental value of $l=47 \mu$ and $\omega=5.74 \times 10^{10} \mathrm{sec}$, we obtain $\sigma=0.745 \times 10^{16}$ $\sec ^{-1}$ (corresponds to a value of resistivity $\rho=121$ $\times 10^{-6} \Omega \mathrm{cm}$ ). This value of $\sigma$ compares favorably with the result of Nigh, Legvold, and Spedding, ${ }^{20}$ in which the resistivity is $119 \times 10^{-6} / \Omega \mathrm{cm}$ along the $c$ axis. In the second step, we note that the ratio of transmitted signal at peak to the signal at region I depends only on the value of relaxation time $\tau$. From the data we obtain $\tau=7.25 \times 10^{-11} \mathrm{sec}$. With the above values of $\sigma$ and $\tau$, each data point can now be associated with a unique value of $M$ through Eq. (8). To obtain $\beta$, we first obtain an approximate value of $T_{C}$ by plotting $\ln M v \sin \left(T_{C}-T\right)$ as shown in Fig. 6. The correct $T_{C}$ should yield roughly a straight line. The slope of the line is $\beta$. To improve the accuracy, we plot $M^{1 / \beta}$ as a function of $T$ (see inset to Fig. 5). The result should be a straight line with the intercept at $M=0$ giving the value of $T_{C}$. Using this new value of $T_{C}$ we plot $\ln M$ vs $\ln \left(T_{C}-T\right)$ again and repeat the process until the best fit to the data gives straight lines on both plots. The above fitting procedure yields

$$
M(T) / M(0)=0.49\left(1-T / T_{C}\right)^{0.31}, \quad T_{C}=295^{\circ} \mathrm{K},
$$

where the value $\beta=0.31$ is the mean of the credible limits indicated by dashed lines on Fig. $6-\beta=0.36$ and $\beta=0.26$. The value $B=0.49$ is the mean of $B$ $=0.52$ and $B=0.46$ (indicated by dashed lines on inset to Fig. 5) where we have used the experimental value of $M(0)=2010 \mathrm{G} .{ }^{21}$ Plotting the data with different $T_{C}$ and $\beta$ indicates that the above limits are generously adequate. The values of $\beta$ and other magnetic critical exponents of Gd are displayed in Table I.

Referring to this Table it seems worthwhile to indicate that our experimental value for $\beta$ is more consistent with the possibility that $\beta$ is $\frac{1}{3}$ or with the Ising model value of 0.312 , than with the equality $\gamma=\beta(\delta-1)$ required by the scaling hypothesis, since using this value of $\beta$ together with other coefficients in the table we obtain $\gamma-\beta(\delta-1)=0.37$ \pm 0.3 . However, the inequality $\gamma>\beta(\delta-1)$ is strictly satisfied as it must be from general thermodynamic arguments (cf. Ref. 1, p. 61, No. 5, and p. 185 , No. 5). We should also comment on the fact that a least-squares fit to our data would produce a considerably smaller error range on our exponent determination, but we refrain from relying on statistical analysis when we are not able to be entirely certain about systematic effects in this completely new method of measurement.

One feature of this method of determining $\beta$ should be noted. In most experiments which determine critical coefficients, the order parameter is measured with the least ambiguity and the greatest precision when one is far from the critical temperature and finally, as one approaches very close to it, the presence of noise and fluctuations causes a degeneration of the signal and a degenera-

TABLE I. Summary of critical exponents for Gd. Definition: $H / H_{1}=E[M / M(0)]^{6}, M(T) / M(0)=B\left(1-T / T_{C}\right)^{\beta}$, $\chi(T) / \chi_{1}=C\left(T / T_{C}-1\right)^{-\gamma}$, where $H_{1}=k T_{C} / m, m$ is the magnetic moment per spin, and $\chi_{1}=M(0) / H_{1}$.

\begin{tabular}{|c|c|c|}
\hline & \multicolumn{2}{|c|}{ Range of $\left(T-T_{C}\right) / T_{C}$} \\
\hline \multirow[t]{2}{*}{$\begin{array}{l}\gamma^{a, b} \\
E\end{array}$} & $\begin{array}{l}1.3 \pm 0.1 \\
0.32\end{array}$ & $2 \times 10^{-3}-2 \times 10^{-1}$ \\
\hline & \multicolumn{2}{|c|}{ Range of $M / M(0)$} \\
\hline $\begin{array}{l}\delta^{a, b} \\
C\end{array}$ & $\begin{array}{l}4.0 \pm 0.1 \\
1.6\end{array}$ & $1 \times 10^{-1}-4 \times 10^{-1}$ \\
\hline C & \multicolumn{2}{|c|}{ Range of $\left(T_{C}-T\right) / T_{C}$} \\
\hline $\begin{array}{l}\beta^{c} \\
B\end{array}$ & $\begin{array}{l}0.31 \pm 0.05 \\
0.49 \pm 0.03\end{array}$ & $3 \times 10^{-3}-5 \times 10^{-2}$ \\
\hline
\end{tabular}

${ }^{2}$ C. D. Graham, Jr., J. Appl. Phys. 36, 1135 (1965).

${ }^{b} \mathrm{P}$. Heller, Rept. Progr. Phys. 30, 784 (1967).

cThis work. 
tion in the measurement of the order parameter. In this experiment, however, it is just in the vicinity of the critical temperature that the most rapid variation of transmitted power occurs, and then farther from the critical point in the region of large magnetization the signal is weaker and noisier because the experiment does not measure the magnetization directly, but rather the change in magnetization through its effect on the transmitted power. Thus good data are to be had near the critical point, but the experiment cannot determine the order parameter over a very great change in magnitude.

Let us now discuss the effects of the domains and the tilting of the $c$ axis $15^{\circ}$ out of the sample plane. Other effects, such as exchange interaction and magnetic anisotropy energy, are discussed in the appendices. It is clear from Sec. II that as long as the signal propagates in a single domain, Eq. (8) holds. However, when there are several domains with the directions of magnetization reversed from one domain to the next it might be expected that there is some loss when signals cross the Bloch walls. This loss can be viewed as a shortening of the relaxation time $\tau$, which simply distributes the loss uniformly across the sample. The tilting of the $c$ axis out of the sample surface means that the transmission mode, instead of propagating into the sample at $90^{\circ}$ with respect to the sample surface, now propagates at $90^{\circ}-15^{\circ}$ (where $15^{\circ}$ is the tilting angle) with respect to the sample surface. The net effect is a loss of signal strength which can again be described by a shortening of $\tau$. We ther efore expect the value $\tau \simeq 7.3$ $\times 10^{-11} \mathrm{sec}$ to be shorter than the true value.

\section{ACKNOWLEDGMENTS}

We wish to thank Dr. F. H. Spedding for providing the sample material and Dr. Paul Schmidt for suggesting the chemical lapping technique. One of us $\left(P_{.} S_{\circ}\right)$ wishes to acknowledge helpful discussions with Dr。T. C. McGill.

\section{APPENDIX A: ROLE OF EXCHANGE INTERACTION IN THEORY}

The Bloch equation with exchange interaction is

$$
\frac{\partial M}{\partial t}=\gamma_{e} \overrightarrow{\mathrm{M}} \times\left(\frac{2 A}{M^{2}} \nabla^{2} \overrightarrow{\mathrm{M}}+\overrightarrow{\mathrm{H}}\right)+\frac{(\overrightarrow{\mathrm{M}})_{\text {transverse }}}{\tau},
$$

where $\nabla^{2} \overrightarrow{\mathrm{M}}$ is the phenomenological exchange interaction term. ${ }^{22}$

The Maxwell's equations are identical to Eq. (4). In component form, the equations are

$$
\begin{aligned}
& \left(k^{2}-2 i / \delta^{2}\right) h_{x}-4 \pi m_{x} 2 i / \delta^{2}=0, \\
& h_{y}=-4 \pi m_{y}, \\
& -i \omega m_{x}=\left(4 \pi M \gamma_{e}-2 k^{2} A \gamma_{e} / M\right) m_{y}-m_{x} / \tau,
\end{aligned}
$$

$$
-i \omega m_{y}=h_{x} M \gamma_{e}+\left(2 A k^{2} \gamma_{e} / M\right) m_{x}-m_{y} / \tau
$$

The equation for $k$ is ${ }^{23}$

$$
\begin{aligned}
& \alpha^{4} k^{6}-\left(2 i \alpha^{2}+1\right) \alpha^{2} k^{4}+ {\left[4 i \alpha^{2}+(\lambda-i \Omega)^{2} / R^{2}\right] k^{2} } \\
&-2 i\left[1+(\lambda-i \Omega)^{2} / R^{2}\right]=0,
\end{aligned}
$$

where $\alpha^{2}=A / 2 \pi M^{2}(0) \delta^{2}$ and $k$ is measured in units of skin depth $\delta$.

The parameter $\alpha^{2}$ is small $\left(\sim 10^{-5}\right.$ for Gd).

Since $\alpha^{2}$ is small, the solutions (in $k^{2}$ ) to (A6) divide naturally into two groups: one solution independent of $\alpha^{2}$ and two solutions with $k^{2} \propto 1 / \alpha^{2}$. In the first case the first two terms of (A6) are negligible and we get from the last two terms

$$
k_{a}^{2} \simeq 2 i\left[1+R^{2} /(\lambda-i \Omega)^{2}\right],
$$

exactly the solution we had before. In the second case the first three terms of (A6) are large since they are proportional to $1 / \alpha^{2}$. The last term becomes negligible. In this approximation we get

$$
k_{b, c}^{2} \simeq \frac{1 \pm\left[1-4(\lambda-i \Omega)^{2} / R^{2}\right]^{1 / 2}}{2 \alpha^{2}} .
$$

It is obvious that $k_{b, c}$ is much larger in magnitude than $k_{a}$, which suggests that the spin-wave modes are strongly damped. To complete the calculation, let us proceed to calculate the coupling of the incident microwaves to the two spin-wave modes. The boundary conditions in this case are ${ }^{23}$ (i) parallel components of electric and magnetic fields are continuous and (ii) $\partial m_{x} / \partial y=0$ and $\partial m_{y} / \partial y=0$ if the axes are defined as in Fig. 1. The equations are

$$
\begin{aligned}
& h_{a}+h_{b}+h_{c}=h_{0}, \\
& k_{a} \frac{R^{2}}{(\lambda-i \Omega)^{2} 4 \pi} h_{a}+k_{b} \frac{g}{8 i \pi \alpha^{2}} h_{b}+k_{c} \frac{f}{8 i \pi \alpha^{2}} h_{c}=0, \\
& k_{a} \frac{R^{2}}{(\lambda-i \Omega) 4 \pi} h_{a}+k_{b} \frac{(i \Omega-\lambda) g}{8 i \pi \alpha^{2}(1-g)} h_{b} \\
& \quad+k_{c} \frac{(i \Omega-\lambda) f}{8 i \pi \alpha^{2}(1-f)} h_{c}=0,
\end{aligned}
$$

where

$$
\begin{aligned}
& g=\frac{1}{2}+\left[\frac{1}{4}-(\lambda-i \Omega)^{2} / R^{2}\right]^{1 / 2}, \\
& f=\frac{1}{2}-\left[\frac{1}{4}-(\lambda-i \Omega)^{2} / R^{2}\right]^{1 / 2} .
\end{aligned}
$$

$h_{0}$ is the magnetic field just outside the sample, and $h_{a}, h_{b}, h_{c}$ are the amplitudes of the magnetic fields associated with the waves $k_{a}, k_{b}, k_{c}$, respectively. $h_{c}$ and $h_{b}$ can be solved in terms of $h_{0}$. For example,

$$
h_{c}=\frac{k_{a} R^{3}}{(\lambda-i \omega)^{2}} \frac{2 i \alpha^{3}}{f^{3 / 2}} \frac{1-f^{2}}{2 f-1} h_{0} .
$$

The amplitudes of the spin-wave modes are proportional to $\alpha^{3}$. Therefore, these modes are negligible in terms of energy transport. 


\section{APPENDIX B: EFFECT OF MAGNETIC ANISOTROPY ENERGY}

Suppose a magnetic field $H$ is applied to the sample along the direction of magnetization. With similar arguments as those in Sec. II, the following equation for the propagation vector is obtained:

$$
k_{2}^{2}=\frac{2 i}{\delta^{2}}\left(1+\frac{R\left(R+\omega_{H}\right)}{\omega_{H}\left(R+\omega_{H}\right)+(\lambda-i \Omega)^{2}}\right) .
$$

Here $\omega_{H}=H / 4 \pi M(0)$ is a dimensionless quantity proportional to the strength of the external field. When $\lambda=0$, we recover from Eq. (B1) the condition for ferromagnetic absorption resonance $\omega_{\text {abs res }}$ $=\gamma_{e}(H B)^{1 / 2}$, at which the imaginary part of $k_{2}$ is maximum. We treat the effect of anisotropy energy as similar to that of applying an external field $H_{A}$ $=2 K_{1} / M(T)$. If we use the experimental value of $K_{1}=1.9 \times 10^{5} \mathrm{erg} / \mathrm{cm}^{3}$ around the Curie point, ${ }^{24}$ a simple calculation shows that anisotropy energy is important when $R \lesssim 0.06$ (at $0.06, \omega_{H} \simeq \Omega$ ). Therefore, only that data point closest to $T_{C}$ is affected. When we take the anisotropy energy into account, that point is raised slightly as indicated by a cross on Fig. 6

\section{APPENDIX C: EFFECT OF MAGNETOSTRICTION}

In theoretical models the exchange constant $J$ is assumed to be constant. But the definition of $J$ contains lattice parameters which vary slowly with temperature. This can be expressed as a slight temperature dependence of the Curie point $T_{C}$ $=T_{c}(T) .^{25}$ Therefore, $M \simeq\left\{\left[T_{c}(T)-T\right] / T_{c}(T)\right\}^{\beta}$, the effect of a temperature varying $T_{C}$ can be expressed as follows:

$$
T_{c}(T) \simeq T_{C^{+}}^{0} \frac{d T_{c}(T)}{d T}\left(T-T_{C}^{0}\right),
$$

where $T_{C}^{0}$ is the experimentally observed Curie temperature.

$$
\begin{aligned}
\frac{T_{c}(T)-T}{T_{C}(T)} & \simeq\left(T_{c}^{0}-T\right)\left(1-\frac{d T_{c}(T)}{d T}\right) / T_{c}^{0}\left(1+\frac{d T_{c}(T)}{d T} \frac{T-T_{C}^{0}}{T_{C}^{0}}\right) \\
& \simeq \frac{T_{c}^{0}-T}{T_{C}^{0}}\left(1-\frac{d T_{c}(T)}{d T}\right)\left(1+\frac{d T_{C}(T)}{d T} \frac{\left(T_{C}^{0}-T\right)}{T_{C}^{0}}\right),
\end{aligned}
$$

where we have assumed $T$ close to $T_{C}^{0}$. From the experimental results ${ }^{26}$

$$
\frac{1}{C} \frac{d C}{d T}=-5 \times 10^{-5}\left({ }^{\circ} \mathrm{K}\right)^{-1},
$$

and $^{27}$

$$
\frac{1}{T_{C}} \frac{d T_{C}}{d p}=-1.18 \times 10^{-5}\left(\mathrm{~kg} / \mathrm{cm}^{2}\right)^{-1}
$$

and $^{28}$

$$
K=2.56 \times 10^{-6}\left(\mathrm{~kg} / \mathrm{cm}^{2}\right)^{-1},
$$

where $C$ is the lattice parameter along the $c$ axis, $p$ is the pressure, and $K$ is the compressibility; it can be estimated that

$$
\frac{1}{C} \frac{d C}{d p} \simeq-0.85 \times 10^{-6}\left(\mathrm{~kg} / \mathrm{cm}^{2}\right)^{-1}
$$

and

$$
\begin{aligned}
\frac{d T_{C}(T)}{d T}=\frac{d T_{C}}{d C} \frac{d C}{d T} & \simeq-0.75 \times 10^{-3} T_{C}\left({ }^{\circ} \mathrm{K}\right)^{-1} \\
& \simeq-0.22 .
\end{aligned}
$$

Therefore,

$$
\begin{aligned}
\left(\frac{T_{C}(T)-T}{T_{C}(T)}\right)^{\beta} \simeq(1.22)^{\beta}\left(\frac{T_{C}^{0}-T}{T_{C}}\right)^{\beta} \\
\times\left[1-0.22\left(\frac{T_{C}^{0}-T}{T_{C}^{0}}\right)\right]^{\beta} .
\end{aligned}
$$

$(1.22)^{\beta}$ can be included in a new value of $B$, and in our present epxeriment, $\left(T_{C}^{0}-T\right) / T_{C}^{0}$ is at most

0.05 . The correction to $\beta$ is therefore negligible.

\section{APPENDIX D: TEMPERATURE GRADIENT ACROSS SAMPLE OWING TO MICROWAVE POWER DISSIPATION}

The sample is mounted between the two cavities with brass plates of thickness $L=\frac{1}{16}$ in. : The microwave power reaches the sample through a coupling hole in the plate of radius $a=\frac{3}{64}$ in. (see Fig. 2 ). The power $P$ that is actually dissipated at the sample is substantially less than the total power present in the excitation cavity as estimated below. If we assume that there is no heat loss by the sample to convection by the air we may use the heatflow equation to find

$$
\frac{d T}{d r}=-\frac{P}{2 \pi a^{2} k l} r, \quad T(r)=-\frac{P}{4 \pi k l} \frac{r^{2}}{a^{2}}+T(0),
$$

where $k$ is the thermal conductivity, $l$ the thickness of the sample, and $T(r)$ the temperature at some distance $r$ from the center. Hence the maximum temperature deviation along the sample is

$$
\Delta T=T(0)-T(a)=P / 4 \pi k l .
$$

We must now estimate $P$. Power dissipation in a cavity is proportional to the area $S$ and the square root of the resistivity $\rho$ of the dissipating 
surface. So $P_{c} / P_{s}=\left(S_{c} / S_{s}\right)\left(\rho_{c} / \rho_{s}\right)^{1 / 2}$ where indices $c$ and $s$ correspond to cavity walls and sample, respectively. For the gadolinium sample used we have $S_{c} \simeq 3.4$ in. $^{2} ; S_{s} \simeq 6.9 \times 10^{-3}$ in. $^{2}$; and $\rho_{c} / \rho_{s}$ $=6.3 / 121$, so $P_{c} / P_{s} \simeq 120$ and $P_{s} \simeq 2 \mathrm{~mW}$ since the total dissipated power $P_{c}+P_{s} \simeq 250 \mathrm{~mW}$.

Using $P=2 \mathrm{~mW}, k \simeq 0.4 \mathrm{~W} / \mathrm{cm}^{\circ} \mathrm{K}$ and $l=50 \times 10^{-4}$ $\mathrm{cm}$ in (D1) we find $\Delta T \simeq 80 \mathrm{~m}^{\circ} \mathrm{K}$ to be the maximum temperature deviation across the sample. Since only heat conduction by the film is considered the above is an overestimate of $\Delta T$ and we may feel confident that the temperature regulation error of $50 \mathrm{~m}^{\circ} \mathrm{K}$ is the dominant limitation in our knowledge of the temperature of the sample.
*Work supported by two grants from the National Science Foundation.

† Present address: Department of Physics, S. U.N.Y., Buffalo, N. Y. 14214.

\$Present address: Institute for Advanced Study, Princeton, N. J. 08540.

${ }^{1} \mathrm{H}$. E. Stanley, Introduction to Phase Transitions and Critical Phenomena, (Oxford U.P., New York, 1971) and references 2 and 5 contain an exhaustive bibliography.

${ }^{2}$ M. E. Fisher, Rep. Prog. Phys. 30, 615 (1967)..

${ }^{3}$ R. J. Baxter, Phys. Rev. Lett. 26, 834 (1971).

${ }^{4}$ P. Heller and G. B. Benedek, Phys. Rev. Lett. $\underline{8}, 428$ (1962).

${ }^{5}$ P. Heller, Rep. Prog. Phys. 30, 731 (1967).

${ }^{6}$ M. Vicenti-Missoni, R. F. Joseph, M. S. Green, and J. M. H. Levelt-Sengers, Phys. Rev. B 1, 2312 (1970).

${ }^{7}$ R. B. Lewis and T. R. Carver, Phys. Rev. Lett. 12, 693 (1964); N. S. VanderVen and R. T. Schumacher, Phys. Rev. Lett. 12, 695 (1964).

${ }^{8}$ G. C. Alexandrakis, O. Horan, and T. R. Carver, Phys. Rev. B 5,3473 (1972).

${ }^{9}$ P. Sheng, Bull. Am. Phys. Soc. 16, 380 (1971); P. Sheng, Ph.D. thesis (Princeton University, 1971) (unpublished), Chap. 2.

${ }^{10}$ A. F. Kip, C. Kittel, A. M. Portis, R. Barton, and F. H. Spedding, Phys. Rev. 89, 518 (1953).

${ }^{11} \mathrm{G}$. C. Alexandrakis, Ph. D. thesis (Princeton University, 1970) (unpublished).

${ }^{12}$ In Eq. (2) in this paper the term $\epsilon \partial E / \partial t$ is neglected because for a typical conductor, $\sigma \sim 10^{7} / \Omega \mathrm{m}$ and $\epsilon \sim 10^{-11}$
$\mathrm{F} / \mathrm{m}$. Therefore $\epsilon \partial E / \partial t$ in Eq. (2) is $\omega \epsilon / 4 \pi \sigma \sim \nu \times 10^{-18}$ or about $10^{-8}$ smaller in magnitude than $4 \pi \sigma E / c$.

${ }^{13}$ R. B. Lewis and T. R. Carver, Phys. Rev. $\underline{155}, 309$ (1967).

${ }^{14} \mathrm{~S}$. Schultz, in Magnetic Resonance and Radiofrequency Spectroscopy, edited by P. Aurbach (North-Holland, Amsterdam, 1969), p. 108.

${ }^{15} \mathrm{~F}$. H. Spedding, Institute for Atomic Research and Dept. of Physics, Iowa State University, Ames, Iowa 50010.

${ }^{16}$ P. H. Schmidt, Bell Telephone Laboratories, Murray Hill, N. J. 07974.

${ }^{17}$ H. E. Nigh, S. Legvold and F. H. Spedding, Phys. Rev. 132,1092 (1963).

${ }^{18}$ C. D. Graha ,, J. Appl. Phys. 36, 1135 (1965).

${ }^{19}$ F. J. Darnell, Phys. Rev. 130, 1825 (1963).

${ }^{20}$ H. E. Nigh, S. Legvold, and F. H. Spedding, Phys. Rev. 132,1092 (1963).

${ }^{21}$ C. Kittel, Introduction to Solid State Physics (Wiley, New York, 1966), p. 461.

${ }^{22}$ S. V. Vonsovskii, Ferromagnetic Resonance (Pergamon, New York, 1966), p. 461.

${ }^{23}$ W. S. Ament and G. T. Rado, Phys. Rev. 97, 1558 (1955).

${ }^{24}$ W. D. Corner, W. C. Roe, and K. N. R. Taylor, Proc. Phys. Soc. 80, 927 (1962).

${ }^{25}$ Peter Heller, Phys. Rev. 146, 403 (1966).

${ }^{26}$ F. J. Darnell, Phys. Rev. 130,1825 (1963).

${ }^{27}$ Lyle Patrick, Phys. Rev. 93, 384 (1954).

${ }^{28} \mathrm{CRC}$ Handbook of Chemistry and Physics, 52nd ed. , edited by F. H. Spedding (Chemical Rubber Co., Cleveland, Ohio, 1971), p. B 233. 